\title{
Clinical outcome of photoactivated platelet-rich plasma in the treatment of knee osteoarthritis
}

\author{
Mohiuddin AKM ${ }^{1}$, Lewis $\mathrm{P}^{2}$, Choudhury $\mathrm{KN}^{3 *}$ and Sadiq BU ${ }^{1}$ \\ ${ }^{1}$ Surecelll Medical Bangladesh Limited, Bangladesh \\ ${ }^{2}$ Surecell Australia, Australia \\ ${ }^{3}$ National Centre for Control of Rheumatic Fever and Heart Disease, Bangladesh
}

\begin{abstract}
Introduction: Osteoarthritis (OA) of the knee is the most common chronic degenerative joint diseases affecting the quality of life of patients. Pain and loss of function are the main clinical features that lead to treatment. For middle-aged and older patients with earlier stages of OA, conservative nonsurgical interventions have been proposed to treat the painful joint. More recently, platelet-rich plasma (PRP), a biological therapy, has become a treatment option to improve the status of the joint for patients with OA. The present study evaluated the clinical outcome of photoactivated platelet-rich plasma (PA-PRP) in patients with chronic knee osteoarthritis.
\end{abstract}

Methods: A total of 232 patients with knee osteoarthritis, aged between 40-70 years were enrolled and treated in the present study and intervention for twelve months. The patients fulfilled the American College of Rheumatology (ACR) criteria for knee OA. The Western Ontario and McMaster Universities OA index (WOMAC) Questionnaires were used for outcome measurement. Results were evaluated after 12 months of intervention.

Results: Female and male patients were $66.8 \%$ and $33.2 \%$ respectively. Mean age and SD of the patients were $55.69( \pm 9.09)$ and $56.19( \pm 9.09)$ respectively. The mean (SD) score of pain, stiffness and physical function during the 1st visit before treatment were $6.74( \pm 3.00), 2.16( \pm 1.85)$ and $22.30( \pm 9.17)$ and after showed $2.44( \pm$ $1.66), 0.60( \pm 0.74)$ and $8.84( \pm 4.20)$ respectively.

The total mean WOMAC scores during the first visit and after treatment were $31.21( \pm 12.19)$ and $11.82( \pm 5.65)$ respectively. The total score of reduction was statistically significant after treatment $(\mathrm{p}=0.00)$.

The principal findings of the study were that the mean WOMAC score reduced and there was improved pain, stiffness and functional capacity of patients with knee OA.

Conclusion: In conclusion, treatment with PA-PRP for patients with knee osteoarthritis produced beneficial effects in terms of clinical outcomes. These results suggested that PA-PRP might be a valuable therapeutic agent for knee osteoarthritis in Bangladesh.

\section{Introduction}

Osteoarthritis is a major cause of knee disability involving cartilage damage related to an inadequate healing response in the inflammatory milieu. Current non-surgical treatment modalities include physiotherapy, analgesia, non-steroidal anti-inflammatory drugs, and intra-articular injections, such as hyaluronic acid, corticosteroid, or Ozone, with the purpose of reducing symptoms and improving joint function [1].

Osteoarthritis (OA) of the knee is one of the most common chronic degenerative joint diseases affecting the quality of life of patients [2]. Knee OA is a degenerative disease that may develop due to aging, trauma, strain, obesity, joint deformity and congenitally abnormal joints [3]. It is caused by many factors such as a degradation of articular cartilage, injury, joint edge and subchondral bone hyperplasia, and ischemia. Knee OA is characterized joint pain, tenderness, stiffness, joint swelling, restricted movement, quadriceps wasting and joint deformity. Pain and loss of function are the main clinical features that lead to treatment $[4,5]$. Although knee-replacement surgery provides an effective solution for severe knee OA [6], for patients with earlier stages of OA, for young patients, for those with co-morbidities and age-related anaesthetic risk, for those who fear surgery, and for those without access to surgery for socio-economic reasons, conservative, nonsurgical interventions have been proposed to treat the painful joint $[7,8]$. Conservative nonsurgical interventions include analgesics, non-steroid and steroidal anti-inflammatory oral medications, and corticosteroid and hyaluronic acid (HA) injections. Although these agents may be beneficial in the short term, there is a lack of evidence that such interventions alter the progression of $\mathrm{OA}$, may worsen longterm outcomes or have side effects. More recently, photoactivated platelet-rich plasma has become a treatment option to improve the status of the joint for patients with OA [9-11].

PRP is an autologous blood product that contains high concentrations of growth factors including vascular endothelial growth factors transforming growth factor- $\beta$, epidermal growth factor, fibroblast growth factor, and platelet-derived growth factor.

*Correspondence to: Choudhury KN, National Centre for Control of Rheumatic Fever and Heart Disease, Bangladesh, Tel: 8801711359459; E-mail: c.kamrun2014@gmail.com

Key words: photoactivated; platelet-rich plasma; knee osteoarthritis; WOMAC score; regenerative medicine

Received: December 03, 2018; Accepted: December 14, 2018; Published: December 17, 2018 
These growth factors serve to promote local angiogenesis, modulate inflammation, inhibit catabolic enzymes and cytokines, recruit local stem cells and fibroblasts to sites of damage or injury and induce healthy nearby cells to manufacture greater numbers of growth factors [12-14]. Thus, the local use of PRP directly at the site of cartilage injury is thought to stimulate a natural healing cascade and accelerate the formulation of cartilage repair tissue $[15,16]$.

We hypothesized that PA-PRP injections would be more efficacious for pain relief and functional improvement in the treatment of patients with knee OA.

- Growth factors help synoviocytes to produce more hyaluronic acid and also increase collagen deposition.

- Growth factors make the matrix more resistance to enzymatic degradation.

\section{Material and Methods}

Study design: A prospective follow up study for twelve months.

Objective: The present study evaluated the clinical outcome of photoactivated platelet-rich plasma in patients with chronic knee osteoarthritis.

Ethics statement: Our research was conducted in accordance with the principal of the Declaration of Helsinki. All patients were required to review trail protocols and subsequently provided their informed consent. All the participants who signed the consent form they were included in the study.

Study participants: A total of 232 patients with knee osteoarthritis, aged between 40-70 years were enrolled in the present study.

The patients fulfilled the American College of Rheumatology (ACR) criteria for knee OA. Inclusion criteria were arthralgia over the previous 3 months with radiologic evidence of articular damage (grades 1-4of Kellgren-Lawrence scale) [17]. Exclusion criteria included age over 70 years, history of immunosuppressive and collagen vascular disorders, history or presence of cancer or malignant disorders any infection or active wound of the knee, recent history of severe trauma to the knee, autoimmune and platelet disorders, use of NSAIDs 2 days before injection, history of knee articular injections of corticosteroids during the previous 3 weeks or use of systemic corticosteroids 2 weeks before PA-PRP injections, haemoglobin measures of less than $10 \mathrm{~g} /$ $\mathrm{dl}$ and platelet counts of less than 150,000 per micro litre, history of vasovagal shock, pregnancy or breast feeding.

The study participants attended a screening visit that included history taking, physical examination, laboratory testing, knee radiography, standing anterior-posterior (AP), lateral and skyline views and a survey of medications and supplements.

Outcome measures: The most widely used condition- specific instruments for the assessment of knee OA is the Western Ontario and McMaster Universities OA index (WOMAC) [18-20]. The WOMAC questionnaire is a widely used tool in studies of arthritis. It includes five items for pain, two items for stiffness and 17 items for assessing functional limitation [21]. Each question is scored from 0 to 5 with higher scores implying worse patient status. We used the WOMAC questionnaire in our present study.

Photoactivated plasma rich protein: PA-PRP work as three steps repair, regeneration and restore of the damage cell. A 10-minute exposure of PA-PRP to monochromatic light prior to injection.
Photoactivation helps growth factor releasing from platelets, increases pro-inflammatory cytokine receptors, IL1 Ra and IL2 Ra, betaendorphin photo modulation has significant benefit in the biomechanics of tissue regeneration and healing process. One of the largest clinical concerns for patients about PRP treatment is exacerbation of pain at the injected area post treatment and photo activation of PRP has been postulated to overcome this.

Intervention: For the process of PA-PRP preparation patients were referred to the laboratory. The PA-PRP processing was done using the SureCell Kit. The SureCell class two PRP tube maintains sterility throughout the entire process and uses a single spin system. In order to prepare PA-PRP with concentrations of three times the average normal values, $16-18 \mathrm{~mL}$ of blood was first collected from the patients' upper limb cubital vein using a $21 \mathrm{G}$ needle and the SureCell PRP kit contains 9:1 ratio Sodium Citrate Anticoagulant. The blood sample was then centrifuged for 10 minutes at $1000 \mathrm{rmp}$ resulting in three layers: the lower layer made up of red blood cells, the intermediate layer is composed of white blood cells, and the upper layer is composed of plasma. The buffy coat layer and the plasma layer were later collected and photo-activated for 10 minutes.

The skin of the injection site was prepped and draped and 7-8ml of PA-PRP was injected under sterile conditions using a $25 \mathrm{G}$ needle through the lateral suprapatellar approach in extended knee position. After 10-15 minutes of rest, patients were asked to actively flex and extend their knees so that the PA- PRP could spread evenly throughout the joint space. Patients received the next injection after 1week, 2weeks and 6 weeks from the first injection. Patients were evaluated 12 months after the intervention.

Statistical analysis: Final data before and after the treatment was imported and analyzed by SPSS 21 version. Continuous variables were reported as the mean with a $95 \%$ confidence interval (CI) and $\mathrm{P}<$ 0.005 was considered to indicate a statistically significant difference. For comparing variables with normal distribution, paired t-test was used. Qualitative variables were expressed with frequency and percent. The differences between before and after treatment were presented in a Box and Whisker diagram where " $\mathrm{X}$ " and "Horizontal line" in the box indicate the "mean" and "median" of the total score respectively.

A total 232 knee osteoarthritis patients were treated by PRP where $66.8 \%$ (155) were female and $33.2 \%$ (77) were male. In the initial visit before and after PRP treatment the mean age and SD of the respondent was $55.69( \pm 9.09)$ and $56.19( \pm 9.09)$ respectively. Body mass index before and after were $30.02( \pm 3.09)$ and $29.21( \pm 3.33)$ respectively and statically significant $(\mathrm{p}=0.00)$ (Table 1$)$.

The initial assessment was done by WOMAC scoring methods. Scoring was done under pain, stiffness and physical function domains. The higher score indicates the more disability. The total score was calculated by the sum of pain, stiffness and physical function. The highest total score was 96 according to the WOMAC scale. The mean (SD) score of pain, stiffness and physical function during the 1st visit before treatment were $6.74( \pm 3.00), 2.16( \pm 1.85)$ and $22.30( \pm 9.17)$ respectively. After 12 months treatment of PA-PRP treatment, the score was calculated again and showed $2.44( \pm 1.66), 0.60( \pm 0.74)$ and 8.84 $( \pm 4.20)$ in terms of pain, stiffness and physical function respectively (Table 2).

The total WOMAC scores during the initial visit and after treatment were $31.21( \pm 12.19)$ and $11.82( \pm 5.65)$ respectively. Total score reduced significantly after treatment with PRP $(\mathrm{p}=0.00)$ (Figure 1). 


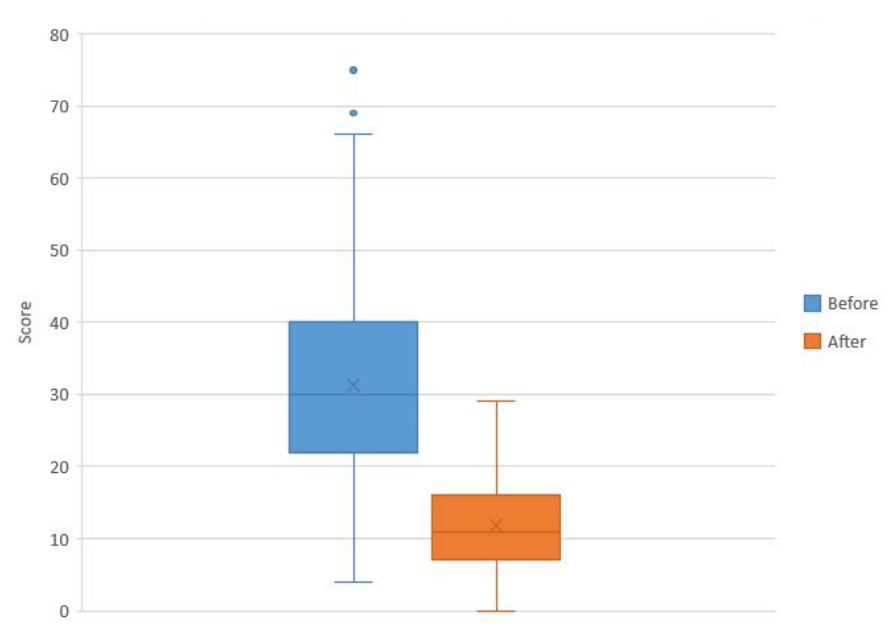

Figure 1. Box and whisker plot of total before and after score (total mean)

Table 1. Baseline characteristics of the study subjects $(\mathrm{N}=232)$

\begin{tabular}{|c|c|c|c|}
\hline \multirow{2}{*}{ Variable } & \multicolumn{2}{|c|}{ Study group } & P-value \\
\cline { 2 - 4 } & $\mathbf{1}^{\text {st }}$ visit & 12-month follow up & \\
\hline Sex & 232 & 232 & - \\
\hline Female (\%) & $155(66.8)$ & $155(66.8)$ & - \\
\hline Male (\%) & $77(33.2)$ & $77(33.2)$ & - \\
\hline Age, mean \pm SD & $55.69 \pm 9.09$ & $56.19 \pm 9.09$ & - \\
\hline BMI, mean \pm SD & $30.02 \pm 3.90$ & $29.21 \pm 3.33$ & 0.00 \\
\hline
\end{tabular}

Table 2. Comparison between before and after the study subject

\begin{tabular}{|c|c|c|c|}
\hline \multirow{2}{*}{ Variable } & \multicolumn{2}{|c|}{ Study group } & \multirow{2}{*}{ P-value } \\
\cline { 2 - 3 } & $\mathbf{1}^{\text {st }}$ visit & $\mathbf{1 2 - m o n t h}$ follow up & \\
\hline WOMAC, mean, SD & \multicolumn{3}{|c|}{} \\
\hline Pain & $6.74 \pm 3.00$ & $2.44 \pm 1.66$ & 0.00 \\
\hline Stiffness & $2.16 \pm 1.85$ & $0.60 \pm 0.74$ & 0.00 \\
\hline Physical Function & $22.30 \pm 9.17$ & $8.84 \pm 4.20$ & 0.00 \\
\hline Total & $31.21 \pm 12.19$ & $11.82 \pm 5.65$ & 0.00 \\
\hline
\end{tabular}

Table 3. Difference of total score in respect to age and sex

\begin{tabular}{|c|c|c|c|c|c|c|c|}
\hline Variables & Number & \multicolumn{2}{|c|}{ 1st visit } & \multicolumn{2}{c|}{$\begin{array}{c}\text { 12-month } \\
\text { Follow-up }\end{array}$} & $\begin{array}{c}\text { Mean } \\
\text { difference }\end{array}$ & p-value \\
\hline $\begin{array}{c}\text { Age } \\
\text { (years) }\end{array}$ & & Mean & SD & Mean & SD & & \\
\hline up to 40 & 14 & 29.64 & 13.64 & 8.79 & 6.42 & 20.85 & - \\
\hline 41 to 50 & 51 & 30.39 & 11.74 & 12.47 & 6.38 & 17.92 & 0.00 \\
\hline 51 to 60 & 95 & 31.15 & 12.57 & 14.26 & 5.26 & 16.89 & - \\
\hline 61 to 70 & 72 & 32.17 & 11.89 & 15.68 & 5.27 & 16.49 & - \\
\hline
\end{tabular}

The total WOMAC score in the different age and gender groups is reduced significantly after PA-PRP treatment of the respondents. In the different age categories, the mean difference showed reduction of $20.85,17.92,16.89$ and 16.49 respectively and statistically significance of $(\mathrm{p}=0.00)$ (Table 3$)$.

\section{Discussion}

A total of 232 patients with knee osteoarthritis aged 40-70years were enrolled for PA-PRP intervention. It was a prospective follow up study for twelve months. The mean age and SD of the respondents for the first visit and after twelve months of PA-PRP treatment were $55.69( \pm 9.09)$ and 56.19 ( \pm 9.09$)$ respectively. Female and male were $66.8 \%(155)$ and $33.2 \%(77)$ respectively. Body mass index before and after were $30.02( \pm 3.09)$ and $29.21( \pm 3.33)$ respectively and statically significant $(\mathrm{p}=0.00)$. The total WOMAC score during the initial visit and after treatment were $31.21( \pm 12.19)$ and $11.82( \pm 5.65)$ respectively. Total score reduced significantly after treatment with PA-PRP $(\mathrm{p}=0.00)$. In the different age categories, the mean reductions in score were 20.85 , $17.92,16.89$ and 16.49 respectively and were statistically significant $(\mathrm{p}=0.00)$.

Currently WHO estimated that $10 \%$ of men and $18 \%$ of women have a painful OA worldwide, and 18 million people of Bangladesh suffering osteoarthritis problem. Our result showed female were suffered more OA of knee than male.

The principal findings of the study show that at twelve months post injection the WOMAC score reduced associated with improved pain, stiffness and functional capacity of patients with knee OA. Our results were similar to the study of Wang- Saegusa, et al. [22] and Seyed Ahmad Raeissadat, et al. [21].

The present author Seyed Ahmad Raeissadat, et al. [21] had previously studied the clinical application of PRP and observed efficacy without adverse events. It was a prospective study published in 2013 on 60 patients treated with two injections of PRP (1 every 4 weeks). Patients underwent clinical evaluation at the beginning and at 6 months follow up. The clinical outcomes revealed a statistically relevant improvement in all the variables of WOMAC and SF-36. In our study we did not work with SF-36,

The systemic review included 14 RCTs and assessed the temporal effect of PRP on knee pain and physical function in the treatment of knee OA compared with other intra-articular injections, including saline, HA, Ozone, and corticosteroid. Data synthesis consistently showed intra-articular PRP injections significantly reduced knee pain, improved physical function and total WOMAC scores compared with control [1]. These reviews showed similar result and reduces WOMAC scores.

PA-PRP is a prominent biomedical blood product that produces efficient outcomes for the treatment of patients with knee osteoarthritis and cartilage disorders [23]. As PA-PRP has been approved as an agent for knee osteoarthritis therapy, it is important for clinicians to understand the clinical indications and outcomes in order to maximize the therapeutic benefits of PA-PRP. The present study revealed that repeated administration of PA-PRP relieved the clinical symptoms of knee osteoarthritis. A previous report indicated that inflammatory cytokines from a complex regulatory signal network in osteonecrosis are mediated by intracellular kinase signalling pathways to regulate recruitment, stimulation and activation of autoimmune cells [24]. Although the causes of knee osteoarthritis are not fully understood, laboratory and clinical evidence have suggested that inflammatory cytokines may contribute to its pathogenesis. Theoretically blocking inflammatory factor pathways may interrupt the inflammatory process and limit joint damage.

Furthermore, the stimulatory effects of PA-PRP treatment have been demonstrated to promote proliferation and chondrogenic differentiation, which may produce beneficial molecules for the maintenance of auricular cartilage [25]. The results of the present study suggest that PA-PRP treatment has a major role to play in the management of OA of the knee in Bangladesh.

\section{Study Limitation}

The limitations of our study were the lack of a control group and the relatively small sample size. The best PA-PRP concentration, long-term effects, the number of injections and the interval between 
treatments and the cost effectiveness of PA-PRP are the issues that necessitate more studies in comparison to control groups and other current treatments. In addition, performing objective studies such as MRI and pathologic assessments would be useful in evaluation the mechanism of action of PA-PRP in patients with OA.

\section{Conclusion}

The principal findings of the study show that at twelve months post injection WOMAC scores reduced with improved pain, stiffness and functional capacity of patients with knee OA.

According to the study result and considering side effects of analgesic and anti-inflammatory medications, PA-PRP injection can be considered a safe and useful therapeutic option in selected patients with mild-to-moderate degrees OA.

In conclusion, treatment with PA-PRP for patients with knee osteoarthritis produced beneficial effects in alleviating joint inflammation, cartilage destruction, bone damage and repairing joint tissue. These results suggested that PA-PRP may be a valuable therapeutic option for knee osteoarthritis.

\section{References}

1. Shen L, Yuan T, Chen S, Xie X, Zhang C (2017) The temporal effect of platelet-rich plasma on pain and physical function in the treatment of knee osteoarthritis: Systemic review and meta-analysis of randomized controlled trials. J Orthop Surg Res 12: 16.

2. Cross M, Smith E, Hoy D, Nolte S, Ackerman I, et al. (2014) The global burden of hip and knee osteoarthritis: estimates from the global burden of disease 2010 study. Ann Rheum Dis 73: 1323-1330. [Crossref]

3. Huang G, Hua S, Yang T, Ma J, Yu W (2018) Platelet-rich plasma shows beneficial effects for patients with knee osteoarthritis by suppressing inflammatory factors. Exp Ther Med 15: 3096-3102.

4. Dai WL, Zhou AG, Zhang H, Zhang J (2017) Efficacy of Platelet-Rich Plasma in the Treatment of Knee Osteoarthritis: A Meta-analysis of Randomized Controlled Trials. Arthroscopy 33: 659-670. [Crossref]

5. Johnson VL, Hunter DJ (2014) The epidemiology of osteoarthritis. Best Pract Res Clin Rheumatol 28: 5-15. [Crossref]

6. Carr AJ, Robertsson O, Graves S, Price AJ, Arden NK, et al. (2012) Knee replacement. Lancet 379: 1331-1340. [Crossref]

7. Bijlsma JW, Berenbaum F, Lafeber FP (2011) Osteoarthritis: an update with relevance for clinical practice. Lancet 377: 2115-2126. [Crossref]

8. Richmond J, Hunter D, Irrgang J, Jones MH, Snyder-Mackler L, et al. (2010) American Acardemy of Orthopaedic Surgeons clinical practice guideline on the treatment of osteoarthritis (OA) of the knee. J Bone Joint Surg Am 92: 990-993.

9. McAlindon TE, bannuruRr, Sullivan MC, Arden NK, Berenbaum F, et al. (2014) OARSI guidelines for the non-surgical management of knee osteoarthritis. Osteoarthritis Cartilage 22: 363-388.
10. Boswell SG, Cole BJ, Sundman EA, Karas V, Fortier LA (2012) Platelet-rich plasma: a milieu of bioactive factors. Arthroscopy 28: 429-439. [Crossref]

11. Sundman EA, Cole BJ, Karas V, Della Valle C, Tetreault MW, et al. (2014) The anti-inflammatory and matrix restorative mechanisms of platelet-rich plasma in osteoarthritis. Am J Sports Med 42: 35-41. [Crossref]

12. Cugat R, Cusco X, seijas R, Álvarez P, Steinbacher G, et al. (2015) Biological enhancement of cartilage repair: The role of platelet-rich plasma and other commercially available growth factors. Arthroscopy 31: 777-783.

13. Supeafico A, Chellini F, Frediani B, Bernardini G, Niccolini S, et al. (2009) Biochemical investigation of the effects of human platelet releasates on human articular chondrocytes. J Cell biochem 108: 1153-1165.

14. Anitua E, Sanchez M, Orive G, Andia I, Andía I (2007) The potential impact of the preparation rich in growth factors (PRGF) in different medical fields. Biomaterials 28: 4551-4560.

15. Kon E, Filardo G, Di Martino A, Marcacci M (2011) Platelet-rich plasma (PRP) to treat sports injuries: evidence to support its use. Knee Surg Sports Traumatol Arthrosc 19: 516-527. [Crossref]

16. Raeissadat SA, Rayegani SM, Babaee M, Ghorbani E (2013) The effect of platelet-rich plasma on pain, function, and quality of life of patients with knee osteoarthritis. Pain Res Treat 2013: 1-7. [crossref]

17. Kohn MD, Sassoon AA, Fernando ND (2016) Classification in Brief: KellgrenLawrence Classification of Osteoarthritis. Clin Orthop Relat Res 474: 1886-1893.

18. Angst F, Aeschlimann A, Steiner W, Stucki G (2001) Responsiveness of the WOMAC osteoarthritis index as compared with the SF-36 in patients with osteoarthritis of the legs undergoing a comprehensive rehabilitation intervention. Ann Rheum Dis 60: 834840 .

19. Bellamy N (1989) Pain assessment in osteoarthritis: experience with the WOMAC osteoarthritis index. Semin Arthritis Rheum 18: 14-17.

20. Stucki G, Meier D, Stucki S, Michel BA, Tyndall AG, et al. (1996) Evaluation of German version of WOMAC (Western Ontario and McMaster Universities) Arthrosis Index. $Z$ Rheumatol 55: 40-49. [Crossref]

21. Raeissadat SA, Rayegani SM, Hassanabadi H, Fathi M, Ghorbani E, et al. (2015) Knee osteoarthritis injection choices: platelet- rich plasma (PRP) versus hyaluronic acid. Clin Med Insights Arthritis Musculoskelet Disord 8: 1-8.

22. Wang-Saegusa A, Cugat R, Ares O, Seijas R, Cuscó X, et al. (2011) Infiltration of plasma rich in growth factors for osteoarthritis of the knee short-term effects on function and quality of life. Arch Orthop Trauma Surg 131: 311-317. [Crossref]

23. Kilincoglu V, Yeter A, Servet E, Kangal M, Yildirim M (2015) Short term results comparison of intraarticular platelet-rich plasma (prp) and hyaluronic acid (ha) applications in early stage of knee osteoarthritis. Int J Clin Exp Med 8: 18807-18812. [Crossref]

24. Gobbi A, Lad D, Karnatzikos G (2015) The effects of repeated intra-articular PRP injections on clinical outcomes of early osteoarthritis of the knee. Knee Surg Sports Traumatol Arthrosc 23: 2170-2177. [Crossref]

25. Görmeli G, Görmeli CA, Ataoglu B, Colak C, Aslantürk O, et al. (2017) Multiple PRP injections are more effective than single injections and hyaluronic acid in knees with early osteoarthritis: a randomized, double-blind, placebo-controlled trial. Knee Surg Sports Traumatol Arthrosc 25: 958-965. [Crossref]

Copyright: (C2018 Mohiuddin AKM. This is an open-access article distributed under the terms of the Creative Commons Attribution License, which permits unrestricted use, distribution, and reproduction in any medium, provided the original author and source are credited. 